\title{
Characterizations for the Dual Split Quaternionic Curves
}

\author{
A. Tuna AKSOY ${ }^{a, *}$ AND A.C. ÇÖKEN ${ }^{b}$ \\ ${ }^{a}$ Süleyman Demirel University, Department of Mathematics, Isparta, Turkey \\ ${ }^{b}$ Akdeniz University, Department of Mathematics, Antalya, Turkey
}

\begin{abstract}
We define harmonic curves and inclined curves for dual split quaternionic curves. And then, we give some characterizations for dual split quaternionic inclined curves by means of the harmonic curvatures.
\end{abstract}

DOI: 10.12693/APhysPolA.132.905

PACS/topics: $02.40 . \mathrm{k}, 02.40 . \mathrm{Hw}$

\section{Introduction}

The quaternions were first described by Hamilton in 1843. In 1987, the Serret-Frenet formulae for quaternionic curves in $E^{3}$ and $E^{4}$ were given by Bharathi and Nagaraj [1], and then, Serret-Frenet formulas for a dual quaternionic curve $D^{3}$ and $D^{4}$ was defined by Sivridağ et al. [2]. Inclined curves and characterization of quaternionic Lorentz manifolds were given by Karadağ [3] and split quaternions were identified with semi-Euclidean space $E_{2}^{4}$, while the vector part of split quaternions was identified with Minkowski 3-space by Inoguchi [4]. After that, Çöken and Tuna in their study [5] have given inclined curves, harmonic curvatures and some characterizations for a quaternionic curve in the semi-Euclidean spaces $E_{2}^{4}$. In 2009, formulas for dual-split quaternionic curves were obtained by Çöken et al. [6].

In this study with the help of Frenet formulas, we give harmonic curvatures and inclined curves for the dual split quaternionic curves. Also, the characterizations are given in terms of harmonic curvatures of curves.

\section{Preliminaries}

A dual number has the form $a+\xi a^{*}$, where $a$ and $a^{*}$ are real numbers and $\xi=(0,1)$ is the dual unit, having property $\xi^{2}=0$. The set of all dual numbers forms a commutative ring over the real number field denoted by Veldkamp [7].

$D^{3}$ dual vector space ( $I D$-module) can be written as $D^{3}=\left\{\left(A_{1}, A_{2}, A_{3}\right): A_{1}, A_{2}, A_{3} \in D\right.$. Similarly, $D^{4}$ dual vector space can be written as $D^{4}=\left\{\left(A_{1}, A_{2}, A_{3}, A_{4}\right)\right.$ : $A_{1}, A_{2}, A_{3}, A_{4} \in D$. The same definitions of innerproduct, norm and cross-product hold for $D^{4}$. The Lorentzian inner-product of two dual vectors $A=a+\xi a^{*}$ and $B=b+\xi b^{*}, a b \in \mathbb{R}_{1}^{3}$ is given as $\langle A, B\rangle=\langle a, b\rangle$ $+\xi\left(\left\langle a^{*}, b\right\rangle+\left\langle a, b^{*}\right\rangle\right)$ with the signature $(-,+,+)$ in $\mathbb{R}_{1}^{3}$. The ID-module $D^{3}$ with the Lorentzian innerproduct is named as semi-dual space $D_{1}^{3}$. On the other hand, a semi-Euclidean inner-product of two dual vectors

*corresponding author; e-mail: abidebytr@yahoo.com in $D^{4}, A=a+\xi a^{*}$ and $B=b+\xi b^{*} ; a, b \in \mathbb{R}_{2}^{4}$, can be defined as

$$
<A, B>=<a, b>+\xi\left(<a^{*}, b>+<a, b^{*}>\right),
$$

with the signature $(-,-,+,+)$ in $\mathbb{R}_{2}^{4}$. The dual space $D^{4}$ with semi-Euclidean inner product is named as semi-dual space $D_{2}^{4}$ or dual-split quaternion $[8,9]$.

A split quaternion $q$ is an expression of the form $q=a e_{1}+b e_{2}+c e_{3}+d$ where $a, b, c$ and $d$ are real numbers, and $e_{1}, e_{2}, e_{3}$ are split quaternionic units which satisfy the non-commutative multiplication rules $Q_{\nu}=\left\{q \mid q=a e_{1}+b e_{2}+c e_{3}+d ; a, b, c, d \in \mathbb{R}\right.$, $\left.e_{1}, e_{2}, e_{3} \in \mathbb{R}_{\nu(\nu=1,2)}^{3}, h_{\nu}\left(e_{i}, e_{i}\right)=-\varepsilon\left(e_{i}\right), 1 \leq i \leq 3\right\}$, where $e_{i} \times e_{i}=-\varepsilon\left(e_{i}\right), 1 \leq i \leq 3, e_{i} \times e_{j}=\varepsilon\left(e_{i}\right) \varepsilon\left(e_{j}\right) e_{k}$ in $\mathbb{R}_{1}^{3}, e_{i} \times e_{j}=-\varepsilon\left(e_{i}\right) \varepsilon\left(e_{j}\right) e_{k}$ in $\mathbb{R}_{2}^{4}$ [5].

A dual split quaternion $Q$ is written as $Q=A e_{1}+$ $\mathrm{Be}_{2}+\mathrm{Ce}_{3}+D$. As a consequence of this definition, a dual split quaternion $Q$ can also be written as $Q=$ $q+\xi q^{*}, \xi^{2}=0$, where $q=a e_{1}+b e_{2}+c e_{3}+d$ and $q^{*}=a^{*} e_{1}+b^{*} e_{2}+c^{*} e_{3}+d^{*}$ are, respectively, real and dual split quaternion components. Let $p$ and $p^{*}$ be two semi-real quaternions.

We define the semi-dual quaternion by $P=p+\xi p^{*}$, and denote the set of semi-dual quaternions by $Q_{D_{\nu}}$ with an index $\nu=1,2$ such, that $Q_{D_{\nu}}=\left\{\left(P \mid P=A e_{1}+B e_{2}+\right.\right.$ $C e_{3}+D ; A, B, C, D \in I D, e_{1}, e_{2}, e_{3} \in \mathbb{R}_{1}^{3}, H_{\nu}\left(e_{i}, e_{i}\right)=$ $\left.\left.-\varepsilon\left(e_{i}\right), 1 \leq i \leq 3\right)\right\}$.

The multiplication of two dual quaternions $P$ and $Q$ is defined by: $P \times Q=p \times q+\xi\left(p \times q^{*}+p^{*} \times q\right)$, where $P=p+\xi p^{*}$ and $Q=q+\xi q^{*}$ and $\times$ shows the quaternion multiplication. It is clear that $P \times Q=S_{P} S_{Q}+$ $S_{P} V_{Q}+S_{Q} V_{P}-<V_{P}, V_{Q}>+V_{P} \Lambda V_{Q}$, where $<,>$ is the inner-product and $\Lambda$ is the cross-product on $I D_{1}^{3}$. The conjugate of $P=S_{P}+V_{P}$ is denoted by $\alpha P=S_{P}-V_{P}$.

For every $P, Q \in Q_{D_{\nu}}$, we define the symmetric dualvalued bilinear form $H_{\nu}: Q_{D_{\nu}} \times Q_{D_{\nu}} \rightarrow I D$ by

$$
\begin{gathered}
H(P, Q)=\frac{1}{2}[\varepsilon(P) \varepsilon(\alpha Q)(P \times \alpha Q)+ \\
\varepsilon(Q) \varepsilon(\alpha P)(Q \times \alpha P)], \text { for } D_{1}^{3}, \\
H(P, Q)=-\frac{1}{2}[\varepsilon(P) \varepsilon(\alpha Q)(P \times \alpha Q) \\
+\varepsilon(Q) \varepsilon(\alpha P)(Q \times \alpha P)], \text { for } D_{2}^{4} .
\end{gathered}
$$


The following results may be obtained:

1) For $\forall P, Q$ of $Q_{D_{\nu}}$, we have $H_{\nu}(P, Q)=h_{\nu}(p, q)+$ $\xi\left[h_{\nu}\left(p, q^{*}\right)+h_{\nu}\left(p^{*}, q\right)\right]$, where $h$ is the symmetric realvalued bilinear form.

2) If $P=A e_{1}+B e_{2}+C e_{3}+D$, then $H_{\nu}(P, P)=$ $-A^{2}-B^{2}+C^{2}+D^{2}$.

3) $\forall P, Q \in Q_{D_{\nu}}$ scalar part and vector part of $P$ are $S_{P}=\frac{1}{2}(P+\alpha P), V_{P}=\frac{1}{2}(P-\alpha P)$.

The concept of a dual spatial quaternion will be used throughout our work. $P$ is called a semi-dual spatial quaternion whenever $P+\alpha P=0$. It is a semi-dual temporal quaternion whenever $P-\alpha P=0$. Let $P$ and $Q$ be two semi-dual spatial quaternions. If $H_{\nu}(P, Q)=0$, then $P$ and $Q$ are $H_{\nu}$-ortogonal [6].

Theorem 2.1. $\beta: I \rightarrow Q_{D_{1}^{3}}$ regular semi-dual quaternionic curve is given by arc-length parameter $s$. Let $\left\{T(s), N_{1}(s), N_{2}(s)\right\}$ be the Frenet trihedron in the point $\beta(s)$ of the curve $\beta$, where $K(s)$ and $R(s)$ are curvatures. Then Frenet apparatus are [6]

$$
\begin{aligned}
& T^{\prime}(s)=\varepsilon_{N_{1}} K(s) N_{1}(s), \\
& N_{1}^{\prime}(s)=\varepsilon_{T}\left[\varepsilon_{T} \varepsilon_{N_{1}} R N_{2}(s)-K T(s)\right], \\
& N_{2}^{\prime}(s)=-\varepsilon_{N_{2}} R N_{1}(s) .
\end{aligned}
$$

Theorem 2.2. $\bar{\beta}: I \rightarrow Q_{D_{2}^{4}}$ regular semi-dual quaternionic curve is given by arc-length parameter $s$. Let $\left\{\bar{T}(s), \bar{N}_{1}(s), \bar{N}_{2}(s), \bar{N}_{3}(s)\right\}$ be the Frenet trihedron in the point $\bar{\beta}(s)$ of the curve $\bar{\beta}$. Then Frenet apparatus are $[6]$,

$$
\begin{aligned}
& \bar{T}^{\prime}(s)=\varepsilon_{\bar{N}_{1}} \bar{K} \bar{N}_{1}, \\
& \bar{N}_{1}^{\prime}(s)=\varepsilon_{N_{1}} K \bar{N}_{2}-\varepsilon_{T} \varepsilon_{\bar{N}_{1}} \bar{K} \bar{T}, \\
& \bar{N}_{2}^{\prime}(s)=-\varepsilon_{T} K \bar{N}_{1}+\varepsilon_{N_{1}}\left[\left(R-\varepsilon_{T} \varepsilon_{\bar{T}} \varepsilon_{\bar{N}_{1}} \bar{K}\right)\right] \bar{N}_{3}, \\
& \bar{N}_{3}^{\prime}(s)=-\varepsilon_{N 2}\left[R-\varepsilon_{T} \varepsilon_{\bar{T}} \varepsilon_{\bar{N}_{1}} \bar{K}\right] \bar{N}_{2} .
\end{aligned}
$$

Definition 2.3. $\beta: I \rightarrow Q_{I D_{1}^{3}}$ regular semi-dual quaternionic curve is given by arc-length parameter $s$, such that $u$ is a constant and unit vector in $Q_{I D_{1}^{3}}$ for $\forall s \in I$, let $H\left(\beta^{\prime}(s), u\right)$ be a constant defined by

$$
\begin{aligned}
& H\left(\beta^{\prime}(s), u\right)=\left\{\begin{array}{c}
\cos \Phi, \beta \text { is spacelike curve } \\
-\cosh \Phi, \beta \text { is timelike curve }
\end{array}\right\}= \\
& \text { const, } \varphi \neq \frac{\pi}{2} .
\end{aligned}
$$

Then curve $\beta$ is called semi-dual spatial quaternionic inclined curve in $Q_{D_{1}^{3}}$

Definition 2.4. Let $\gamma: I \rightarrow \mathbb{R}_{1}^{3}$ be a semi-real quaternionic curve. Such that $\gamma(s)=\gamma_{1}(s) e_{1}+\gamma_{2}(s) e_{2}+\gamma_{3}(s) e_{3}$, $\beta: I \rightarrow Q_{D_{1}^{3}}$

$$
\begin{array}{r}
\beta(s)=\gamma_{1}(s) e_{1}+\gamma_{2}(s) e_{2}+\gamma_{3}(s) e_{3} \\
+\xi\left(\gamma_{1}^{*}(s) e_{1}+\gamma_{2}^{*}(s) e_{2}+\gamma_{3}^{*}(s) e_{3}\right)
\end{array}
$$

or $\beta(s)=A(s) e_{1}+B(s) e_{2}+C(s) e_{3} ; A(s), B(s), C(s) \in$ $I D$, obtained from $\gamma$, such that $\left\{T(s), N_{1}(s), N_{2}(s)\right\}$ be the Frenet trihedron in the point $\beta(s)$ of the curve $\beta$ and let $u=u_{0}+\xi u_{0}^{*}$ be a unit and constant vector. Then, $\beta^{\prime}(s)=T_{0}(s)+\xi T_{0}^{*}(s)=T(s) \alpha \beta^{\prime}(s)=$
$-T_{0}(s)-\xi T_{0}^{*}(s), u=u_{0}+\xi u_{0}^{*}, \alpha u=-u_{0}-\xi u_{0}^{*}(u$ is semi-dual spatial quaternion). Thus, $u, T(s) \in Q_{I D_{1}^{3}}$

$$
H(u, T)=h\left(u_{0}, T_{0}\right)+\xi\left[h\left(u_{0}, T_{0}^{*}\right)+h\left(u_{0}^{*} T_{0}\right)\right] .
$$

Definition 2.5. $\beta: I \rightarrow Q_{D_{1}^{3}}$ regular semi-dual quaternionic curve is given by arclength parameter $s$. Let $\left\{T(s), N_{1}(s), N_{2}(s)\right\}$ be the Frenet trihedron in the point $\beta(s)$ of the curve $\beta$ and let $u=u_{0}+\xi u_{0}^{*}$ be a unit and constant vector, such that angle $\Phi=\varphi+\xi \varphi^{*}$ is between $\beta^{\prime}(s)$ and $u$. $H: I \rightarrow I D$,

$$
\begin{aligned}
& H\left(N_{2}(s), u\right)=\bar{H}(s) H(T(s), u)= \\
& \left\{\begin{array}{c}
\bar{H} \cos \Phi, \beta \text { is a spacelike curve } \\
-\bar{H} \cosh \Phi, \beta \text { is a timelike curve }
\end{array}\right\}, \varphi \neq \frac{\pi}{2}
\end{aligned}
$$

can be defined. Then function $H$ is semi-dual harmonic curvature in the point $\beta(s)$ of curve $\beta$ with respect to $u$.

Definition 2.6. $\bar{\beta}: I \rightarrow Q_{D_{2}^{4}}$ regular semi-dual quaternionic curve is given by arclength parameter $s$, such that $u=u_{0}+\xi u_{0}^{*}$ is a unit and constant semi-dual spatial quaternion for every $s \in I$,

$$
\begin{aligned}
& H\left(\bar{\beta}^{\prime}(s), u\right)=\left\{\begin{array}{c}
\cos \Phi, \bar{\beta} \text { is a spacelike curve } \\
-\cosh \Phi, \bar{\beta} \text { is a timelike curve }
\end{array}\right\}= \\
& \text { const, } \varphi \neq \frac{\pi}{2} .
\end{aligned}
$$

Then $\bar{\beta}$ is called semi-dual quaternionic inclined curve in semi-quaternion sets $Q_{D_{2}^{4}}$.

Definition 2.7. $\bar{\beta}: I \rightarrow Q_{D_{2}^{4}}$ regular semi-dual quaternionic curve is given by arc-length parameter $s$. Let $\left\{\bar{T}(s), \bar{N}_{1}(s), \bar{N}_{2}(s), \bar{N}_{3}(s)\right\}$ be the Frenet apparatus and let $u$ be a unit and constant, such that angle $\Phi=\varphi+\xi \varphi^{*}$ is between $T(s)$ and $u$. Let $\bar{H}_{i}: I \rightarrow I D$ be functions defined by

$$
\begin{aligned}
& H\left(\bar{N}_{i+1}, u\right)=\left\{\begin{array}{c}
\bar{H}_{i} \cos \Phi, \bar{\beta} \text { is a spacelike curve } \\
-\bar{H}_{i} \cosh \Phi, \bar{\beta} \text { is a timelike curve }
\end{array}\right\}, \\
& \varphi \neq \frac{\pi}{2} .
\end{aligned}
$$

Then function $\bar{H}_{i}$ is called $i$ th Harmonic curvature in the point $\bar{\beta}(s)$ of the $\bar{\beta}$ semi-dual quaternion curve with respect to $u$. Then, $\bar{H}_{0}$ is equal to zero.

\section{Harmonic curvatures and characterizations in $D_{1}^{3}$}

Theorem 3.1. Let $\beta: I \rightarrow Q_{D_{1}^{3}}$ be a semi-dual spatial quaternionic inclined curve given by arc-length parameter $s$. Curvatures at the point $\beta(s)$ of $\beta$ are $K(s)=k(s)+\xi k^{*}(s), R(s)=r(s)+\xi r^{*}(s)$ and in that case $\bar{H}$ is a harmonic curvature, it is

$$
\bar{H}(s)=\frac{K(s)}{\varepsilon_{T} \varepsilon_{N_{1}} R(s)}
$$

(see [3] for dual quaternionic curve).

Proof. Let $\Phi=\varphi+\xi \varphi^{*}$ be an angle between the $u$ constant semi-dual vector and the tangent vectors of $\beta: I \rightarrow Q_{I D_{1}^{3}}$ semi-dual spatial quaternionic inclined line, such that $\left\{T(s), N_{1}(s), N_{2}(s)\right\}$ is Frenet trihedron in the point $\beta(s)$. We obtain, that $H(T(s), u)=$ const. 
Here, by differentiating with respect to $s$, we get $H\left(T^{\prime}(s), u\right)=0$. From Serret-Frenet formulas of $\beta$, we obtain that $H\left(N_{1}(s), u\right)=0$. Here, by differentiating with respect to $s$, we obtain $H\left(N_{1}^{\prime}(s), u\right)=0$. Here, by using the Frenet formulas, $H\left(N_{2}(s), u\right)=$ $\frac{\varepsilon_{T} K}{\varepsilon_{T}^{2} \varepsilon_{N_{1}} R} H(T(s), u)$ is obtained. Thus, if Eq. (6) is used, Eq. (9) is found. Thus, harmonic curvature of semi-dual spatial quaternionic curve is obtained from the curvatures of a curve.

Theorem 3.2. Let $\beta: I \rightarrow Q_{D_{1}^{3}}$ be a semi-dual spatial quaternionic curve, given by arc-length parameter $s$. Let $\bar{H}(s)$ be harmonic curvature and $\left\{T(s), N_{1}(s), N_{2}(s)\right\}$ be the Frenet trihedron at the point $\beta(s) . \quad \beta$ is semi-dual quaternionic inclined curve if and only if $\bar{H}^{2}(s)$ is constant (see [3] for dual spatial quaternionic curve).

Proof. $(\Rightarrow)$ There is a unit semi-dual spatial quaternion $u$. Therefore, $H\left(\beta^{\prime}(s), u\right)=0$ is constant for $\beta$ semi-dual spatial quaternionic inclined curve with respect to arc-length parameter $s$. If $\left\{T(s), N_{1}(s), N_{2}(s)\right\}$ is basis of semi-dual spatial quaternion in the point $\beta(s)$, semi dual quaternion $u$,

$$
\begin{aligned}
u & =\varepsilon_{T} H(T(s), u) T(s) \\
& +\sum_{i=1}^{2} \varepsilon_{N_{i}} H\left(N_{i}(s), u\right) N_{i}(s)
\end{aligned}
$$

is obtained. Hence, we have $\|u\|^{2}=|H(u, u)|=$ $\left|\varepsilon_{u}(u \times \alpha u)\right|$. With Eq. (6), by taking $\|T(s)\|=\left|\varepsilon_{T}\right|$, $\left\|N_{1}(s)\right\|=\left|\varepsilon_{N_{1}}\right|,\left\|N_{2}(s)\right\|=\left|\varepsilon_{N_{2}}\right|$ and $\|u\|=\left|\varepsilon_{u}\right|=1$ into consideration,

$$
\bar{H}^{2}(s)=\frac{ \pm 1-H^{2}(T(s), u) \varepsilon_{T}}{H^{2}(T(s), u) \varepsilon_{N 2}}=\mathrm{const}
$$

is obtained.

$(\Leftarrow)$ In contrast, suppose that $\bar{H}^{2}(s)=a$ is constant for $\beta$ semi-dual spatial quaternionic curve. Therefore, there is $\Phi$ angle such that $\frac{ \pm 1-H^{2}(T(s), u) \varepsilon_{T}}{H^{2}(T(s), u) \varepsilon_{N_{2}}}=a$. Thus, we define $u$ semi-dual spatial quaternion, where

$$
\begin{aligned}
u & =\varepsilon_{T} H(T(s), u) T(s) \\
& +\varepsilon_{N 2} \bar{H}(s) H(T(s), u) N_{2}(s) .
\end{aligned}
$$

By differentiating Eq. (11) with respect to $s$

$$
\begin{aligned}
& \frac{\mathrm{d} u}{\mathrm{~d} s}=\varepsilon_{T} H(T(s), u) K(s) N_{1}(s) \\
& \quad-\varepsilon_{N 2}^{2} H(T(s), u) \frac{K(s)}{\varepsilon_{T} \varepsilon_{N_{1}} R(s)} N_{1}(s) .
\end{aligned}
$$

Here, using the Frenet formulas and by taking Eq. (9) into the consideration $\frac{\mathrm{d} u}{\mathrm{~d} s}=0$ is obtained. Thus $u$ is a constant semi-dual spatial quaternion. Now, if it is given that $u$ semi-dual spatial quaternion is a unit,

$$
\begin{aligned}
& \|u\|^{2}=|H(u, u)|=\left|\varepsilon_{u}(u \times \alpha u)\right|= \\
& \quad\left|\varepsilon_{u} H^{2}(T(s), u)\left[\varepsilon_{T}+\bar{H}^{2}(s) \varepsilon_{N^{2}}\right]\right|=\left|\varepsilon_{u}( \pm 1)\right|=1
\end{aligned}
$$

is found. On the other hand, $H(T(s), u)=$ const is found. Therefore, $\beta$ is a semi-dual spatial quaternionic inclined curve.

\section{Harmonic curvatures and characterizations in $D_{2}^{4}$}

Theorem 4.1. Let $\beta: I \rightarrow Q_{D_{1}^{3}}$ be semi-dual spatial quaternionic inclined curve. Such that $\beta(s)=A(s) e_{1}+$ $B(s) e_{2}+C(s) e_{3} ; A(s), B(s), C(s) \in I D . \bar{\beta}(s)=A(s) e_{1}+$ $B(s) e_{2}+C(s) e_{3}+D(s), D(s) \in I D$ is obtained from $\beta$. Thus, semi-dual quaternionic curves $\bar{\beta}$ and $\beta$ are semidual quaternionic inclined curves of the same axis (see [4] for dual quaternionic curves).

Proof. Let $\bar{\beta}: I \rightarrow Q_{D_{2}^{4}}$ be a semi-dual quaternionic curve given by arc-length parameter $s$. Let $u$ be an unit and a constant semi-dual spatial quaternion, such that $\left\{\bar{T}(s), \bar{N}_{1}(s), \bar{N}_{2}(s), \bar{N}_{3}(s)\right\}$ is Frenet apparatus in the point $\bar{\beta}(s)$ of curve $\bar{\beta}$. As we know, $H\left(\bar{\beta}^{\prime}(s), u\right)=H(\bar{T}(s), u), \bar{T}(s)=D(s)+T(s, D(s))=$ $d_{0}+\xi d_{0}^{*} \in I D, T(s)=T_{0}(s)+T_{0}^{*}(s), u=u_{0}+\xi u_{0}^{*}$ and $\alpha T(s)=D(s)-T(s)$. We obtained that

$$
\begin{aligned}
& H(\bar{T}(s), u)= \\
& \left.\quad-\varepsilon_{\bar{T}} \varepsilon_{\alpha u} h\left(u_{0}, T_{0}\right)+\xi\left[h\left(u_{0}, T_{0}^{*}\right)+h\left(u_{0}^{*}, T_{0}\right)\right]\right\},
\end{aligned}
$$

where $h$ is a real semi-quaternionic inner product.

Theorem 4.2. Let $\bar{\beta}: I \rightarrow Q_{D_{2}^{4}}$ be a semi-dual quaternionic inclined curve given by arc-length parameter $s . k_{i}(s)$ are curvatures in the point $\bar{\beta}(s), \bar{\delta}_{i}(s)=\frac{1}{k_{i}(s)}$, $1 \leq i \leq 3$, are curvature radii and $\bar{H}_{j}(s), j=1,2$ are harmonic curvatures,

$$
\begin{aligned}
& \bar{H}_{1}(s)=\frac{1 \text { st curvature }}{2 \text { nd curvature }}, \\
& \quad \text { and } \bar{H}_{2}(s)=\bar{H}_{1}^{\prime}(s) \bar{\delta}_{3}(s),
\end{aligned}
$$

(see [3] for dual quaternionic curves).

Proof. Let $\bar{\beta}: I \rightarrow Q_{D_{2}^{4}}$ be a regular semi-dual quaternionic curve. $u$ is an unit and a constant semi-dual spatial quaternion. If $\left\{\bar{T}(s), \bar{N}_{1}(s), \bar{N}_{2}(s), \bar{N}_{3}(s)\right\}$ is Frenet apparatus in the point $\bar{\beta}(s), H(\bar{T}(s), u)=$ const is written. By differentiating this equation with respect to $s$, we obtain that $H\left(\bar{T}^{\prime}, u\right)=0$. Here, using Eq. (1), $H\left(\bar{N}_{1}, u\right)=0$ is found. By differentiating $H\left(\bar{N}_{1}, u\right)=0$ with respect to $s, H\left(\bar{N}_{1}^{\prime}(s), u\right)=0$ is obtained. Here, using Eq. (2),

$$
H\left(\bar{N}_{2}, u\right)=\frac{\varepsilon_{\bar{N}_{1}} \varepsilon_{T} \bar{K}}{\varepsilon_{N_{1}} K} H(\bar{T}(s), u)
$$

is found. Here, by taking Eqs. (7) and (8) into consideration, $\bar{H}_{1}=\frac{\varepsilon_{\bar{T}} \varepsilon_{\bar{N}_{1}} \bar{K}}{\varepsilon_{N_{1}} K}=\frac{1 \text { st curvature }}{2 \text { nd curvature }}$ is obtained. Here, $\bar{K}$ is the first curvature of $\bar{\beta}$ curve in $Q_{D_{2}^{4}}, K(s)$ is both the first curvature of $\beta$ curve in $Q_{D_{1}^{3}}$ and the second curvature of $\bar{\beta}$ curve in $Q_{D_{2}^{4}}$. On the other hand, if derivative of Eq. (13) with respect to $s$ is taken,

$$
H\left(\bar{N}_{2}^{\prime}, u\right)=\bar{H}_{1}^{\prime} H(\bar{T}(s), u)
$$

is found. Here, using Eq. (3)

$$
\begin{aligned}
& -\varepsilon_{T} K H\left(\bar{N}_{1}, u\right)+\varepsilon_{N_{1}}\left[R-\varepsilon_{T} \varepsilon_{\bar{T}} \varepsilon_{\bar{N}_{1}} \bar{K}\right] H\left(\bar{N}_{3}, u\right)= \\
& -\bar{H}_{1}^{\prime} \cosh \Phi
\end{aligned}
$$

is found. If Eq. (8) is written in place of Eq. (15), $\bar{H}_{2}(s)=\bar{H}_{1}^{\prime}(s) \bar{\delta}_{3}(s)$ is found. Thus, harmonic curvatures for semi-dual spatial quaternionic inclined curves are obtained from curvatures. 
Theorem 4.3. Let $\bar{\beta}: I \rightarrow Q_{D_{2}^{4}}$ be semi dual quaternionic inclined curve, given by arc-length parameter $s$. If $\left\{\bar{T}(s), \bar{N}_{1}(s), \bar{N}_{2}(s), \bar{N}_{3}(s)\right\}$ is Frenet apparatus and harmonic curvature $H_{i}(s), i=1,2$, (see [3] for dual quaternionic curve), $\bar{\beta}$ is a inclined line if and only if $\sum_{i=1}^{2} \varepsilon_{\bar{N}_{i+1}} H^{2}\left(\bar{N}_{i+1}(s), u\right)$ is a constant.

Proof $(\Rightarrow)$ : Suppose that $\bar{\beta}: I \rightarrow Q_{D_{2}^{4}}$ is an inclined line of semi-dual quaternionic curve. Thus, there is $u$ unit and constant semi-dual quaternion such that $H\left(\bar{\beta}^{\prime}(s), u\right)=$ const, $\forall s \in I$ for $\bar{\beta}$ curve. Suppose that there is a basis of $\bar{\beta}$ curve in the point $\bar{\beta}(s)$. We define the semi-dual quaternion $u$ as

$$
\begin{aligned}
u & =\varepsilon_{\bar{T}} H(\bar{T}(s), u) \bar{T}(s) \\
& +\sum_{i=1}^{3} \varepsilon_{\bar{N}_{i}} H^{2}\left(\bar{N}_{i}(s), u\right) \bar{N}_{i}(s) .
\end{aligned}
$$

We have $\|u\|^{2}=|H(u, u)|=\left|\varepsilon_{u}(u \times \alpha u)\right|=1$. Because of $\|\bar{T}(s)\|=\left|\varepsilon_{\bar{T}}\right|,\left\|\bar{N}_{1}(s)\right\|=\left|\varepsilon_{\bar{N}_{1}}\right|,\left\|\bar{N}_{2}(s)\right\|=\left|\varepsilon_{\bar{N}_{2}}\right|$, $\left\|\bar{N}_{3}(s)\right\|=\left|\varepsilon_{\bar{N}_{3}}\right|,\|u\|=\left|\varepsilon_{u}\right|=1$,

$$
\begin{aligned}
& \sum_{i=1}^{2} \varepsilon_{\bar{N}_{i+1}} H^{2}\left(\bar{N}_{i+1}(s), u\right)= \\
& \quad \pm 1-\varepsilon_{\bar{T}} H^{2}(\bar{T}(s), u)=\mathrm{const}
\end{aligned}
$$

is obtained.

$(\Leftarrow)$ Suppose that $\sum_{i=1}^{2} \varepsilon_{\bar{N}_{i+1}} H^{2}\left(\bar{N}_{i+1}(s), u\right)=a$ is a constant for $\bar{\beta}: I \rightarrow Q_{I D_{2}^{4}}$ semi-dual quaternion curve. Therefore, there is a dual angle $\Phi$, such that $\pm 1-\varepsilon_{\bar{T}} H^{2}(\bar{T}(s), u)=a$. Thus, we defined that $u$ is semi-dual spatial quaternion, such that

$$
\begin{aligned}
u & =\varepsilon_{\bar{T}} H(\bar{T}(s), u) \bar{T}(s) \\
& +\sum_{i=2}^{3} \varepsilon_{\bar{N}_{i}} \bar{H}_{i-1}(s) H(\bar{T}(s), u) \bar{N}_{i}(s) .
\end{aligned}
$$

Here, we demonstrate that $u$ is a constant. Thus, if derivative of Eq. (17) with respect to $s$ is taken, and here, using Eq. (8) for $i=1,2, H\left(\bar{N}_{2}(s), u\right)=\bar{H}_{1} H(\bar{T}(s), u)$ and $H\left(\bar{N}_{3}(s), u\right)=\bar{H}_{2} H(\bar{T}(s), u)$ is obtained. If derivative of the last equation with respect to $s$ is taken, $H\left(\bar{N}_{3}^{\prime}(s), u\right)=\bar{H}_{2}^{\prime} H(\bar{T}(s), u)$ is found. Here, using Eq. (4),

$$
-\varepsilon_{N_{2}}\left(R-\varepsilon_{T} \varepsilon_{\bar{T}} \varepsilon_{\bar{N}_{1}} \bar{K}\right) H\left(\bar{N}_{2}, u\right)=\bar{H}_{2}^{\prime} H(\bar{T}(s), u)
$$

is obtained. Here, by considering the $H\left(\bar{N}_{2}(s), u\right)=$ $\bar{H}_{1} H(\bar{T}(s), u)$

$$
\bar{H}_{2}^{\prime}(s)=-\varepsilon_{N_{2}}\left(R-\varepsilon_{T} \varepsilon_{\bar{T}} \varepsilon_{\bar{N}_{1}} \bar{K}\right) \bar{H}_{1}(s)
$$

is obtained. By taking the derivative of Eq. (17) and using the last equations, $\frac{\mathrm{d} u}{\mathrm{~d} s}=0$ is found. Thus, $u$ is a constant. On the other hand, we demonstrate that $u$ is an unit. That is, $\|u\|^{2}=|H(u, u)|=\left|\varepsilon_{u}(u \times \alpha u)\right|=1$ is obtained. Thus, $H(\bar{T}(s), u)=$ const is found. Therefore, $\bar{\beta}$ is an inclined curve.
Corollary 4.4. Derivative equations of harmonic curvatures obtained for semi-dual quaternionic curves by the aid of Eq. (18) and the Theorem (4.2.),

$$
\left[\begin{array}{c}
\bar{H}_{1}^{\prime} \\
\bar{H}_{2}^{\prime}
\end{array}\right]=\left[\begin{array}{cc}
0 & \varepsilon_{N_{1}} F \\
-\varepsilon_{\bar{N}_{2}} F & 0
\end{array}\right]\left[\begin{array}{c}
\bar{H}_{1} \\
\bar{H}_{2}
\end{array}\right]
$$

is found in the matrix form, where $F=\left(R-\varepsilon_{T} \varepsilon_{\bar{T}} \varepsilon_{\bar{N}_{1}} \bar{K}\right)$.

\section{Conclusions}

In this paper, we have studied the differential geometry of smooth curves in the semi-dual spaces $D_{1}^{3}$ and $D_{2}^{4}$. We gave new characterizations for dual quaternionic curves in the semi-dual spaces $D_{1}^{3}$ and $D_{2}^{4}$ using their harmonic curvature functions. These functions are

(i) $\beta: I \rightarrow Q_{D_{1}^{3}}$, which is a semi-dual quaternionic inclined curve if and only if $\bar{H}^{2}(s)$ is constant. Here $\bar{H}$ is harmonic curvature function of the curve $\beta$.

(ii) $\bar{\beta}$ is an inclined line if and only if $\sum_{i=1}^{2} \varepsilon_{\bar{N}_{i+1}} H^{2}\left(\bar{N}_{i+1}(s), u\right)$ is a constant. Here $\bar{H}_{1}$ and $\bar{H}_{2}$ are harmonic curvature functions of the curve $\bar{\beta}$.

\section{Acknowledgments}

This study was supported by the SDU Funding Agency under Grant 2059-D-09.

\section{References}

[1] K. Bharathi, M. Nagaraj, Indian J. Pure Appl. Math. 18, 507 (1987).

[2] A.İ. Sivridă̆, R. Güneş, S. Keleş, Mechan. Mach. Th. 29, 749 (1994).

[3] M. Karada $\breve{g}$, Ph.D. Thesis, Malatya, Turkey 1999.

[4] J. Inoguchi, Tokyo J. Math. 21, 140 (1998).

[5] A.C. Çöken, A. Tuna, Appl. Math. Computation 155, 373 (2004).

[6] A.C. Çöken, C. Ekici, İ. Kocayusufoğlu, A. Görgülü, Kuwait J. Sci. Engin. 35, 21 (2009).

[7] G.R. Veldkamp, Mech. Mach. Th. 11, 141 (1976).

[8] B. O'Neill, Semi-Riemannian geometry with applications to relativity, Academic Press, London 1983.

[9] H.H. Uğurlu, A. Çalışkan, Math. Comput. Appl. 1, 142 (1996). 